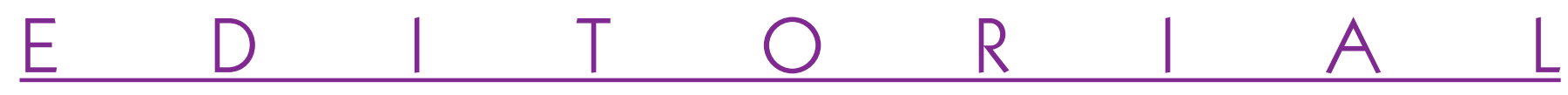

\title{
Etiological treatment of OSA
}

\author{
Jacques TALMANT, Jean-Claude TALMANT, \\ Joël DENIAUD, Philippe AMAT
}

At the end of the last century orthodontists began to become aware of "Obstructive Sleep Apnea (OSA)". And, over the past thirty years, research in this area has flourished, profoundly transforming the quality of life for patients who suffer from this syndrome, with dentofacial orthopedists actively participating in its symptomatic treatment. But, among the issues that remain incompletely elucidated, the actual cause of the snoring episodes that destabilize the pharynx still occupies an important position.

In an article dealing with "Ventilation and mechanics of the oropharynx" published in this journal ten years ago ${ }^{16}$, we proposed a mechanism that could account for the unanticipated arrival of OAS. And we suggested a therapeutic approach, that we had already tested on children and adolescent, that was designed to treat pharyngeal destabilization during sleep. In its ensemble, it is based on:

- the specifically human post-natal anatomic development of the oropharynx (other primates do not suffer from OAS);
- the physiological changes that accompany this development (the absence of an oropharynx in neonates is a morphological blessing that protects the newborn when its ventilatory fluid abruptly becomes aerated at a time when the functional coordination of the pharynx is still adapted to life in the amniotic milieu);

- the characteristics of the homeostatic regulation of its lumen;

- the mechanism of OSA breakdown;

- the possibility of helping current or potential OSA patients with "adaptive" maxillary expansion that can assist patients in recovering physiological control of their pharynxes during nighttime sleeping as well as during the day.

The message of our essay was that dentofacial orthopedists should contribute to the etiological treatment of OSA. Utilized at the proper time, "adaptive" maxillary expansion is an effective, tested clinical procedure capable of correcting the transverse deficit of the nasal fossa and the nasal valves still narrower. Supplemented whenever indicated with immunoallergical 
treatments of the nasal mucosa, and, in certain cases, surgical intervention to correct septal and/or turbinal anomalies, palatal expansion helps to eliminate the causes of snoring and optimizes the framework for nasal

\section{1 - A SHORT STATE OF THE ART}

Since 1993, the American National Center on Sleep Disorders Research, a division of the National Heart Lung and Blood Institute, has been mandated by the National Institutes of Health to conduct and support research on sleep and sleep disorders. According to this organization, Sleep-Disordered Breathing (SDB) is a category that encompasses all qualitative (pauses) or quantitative anomalies of night time breathing. OSA, the most common of these problems, is characterized by repeated complete or partial collapse of the pharynx during sleep, which forces the patient to awaken in order to resume respiration. These frequent interruptions of nightly sleep provoke periods of daytime somnolence and a decrease in the quality of cognitive performance. Associated as they are with intermittent hypoxia and hypercapnia, repeated episodes of awakening are implicated in the development of cardiovascular complications. In addition, there is evolving evidence that SDB may contribute to insulin resistance and other components of the metabolic syndrome.

Despite considerable progress in polysomnography, an essential tool for detection of SDB, most patients remain undiagnosed; and the efficient symptomatic therapeutic approach, breathing as it restores proper function of the soft palate and of the phar$y n x$. In this way patients benefit from a restoration of correct nocturnal breathing. Where do we stand today in dealing with OSA?

continuous positive airway pressure, remains somewhat cumbersome, worse still must be repeated every night for life and hence is not associated with optimal compliance rates. We continue to have a very incomplete understanding of the neurobiological mechanisms responsible for the sleepinduced changes in upper airway motor control that lead to pharyngeal collapse. And we still do not know to what extent SDB in the elderly represents the same disorder as is encountered in younger populations and thus deserves similar therapy.

Snoring, a symptom of increased upper airway resistance during sleep, is extremely frequent in childhood, and affects infants as well as older children. The pathophysiology of SDB in children as in adults is still poorly understood. While adenotonsillar hypertrophy is certainly a major contributor to SDB, others factors such as obesity, craniofacial genetics, and neural control mechanisms of upper air-way patency also appear to be important 6,12 . In addition, the extend of problems endured by children with SDB enlarges progressively, having now recognized cardiovascular and neurobehavioral consequences. These morbidities include pulmonary hypertension, arterial hypertension, nocturnal 
enuresis, reduced somatic growth, learning and cognitive deficits, and behavioral problems that resemble attention deficit-hyperactivity disor- der. A report presented to the Société Française d'Orthopédie Dento-Faciale in $1982^{15}$ identified some of these associations.

\section{2 - PHARYNGEAL TRAUMA RELATED TO SNORING}

Many clinicians have come to believe that SDB are not as innocuous as previously thought, especially in view of the observation that patients begin snoring long before they display indications of obstructive symptoms. In fact, the intensity of the snoring draws attention to the harmful effect of the stresses the mechanical energy of these vibrations apply on the pharyngeal soft tissues ${ }^{1,4,16}$. Driven every night by the turbulent flow of the nasopharyngeal inspiratory air and transmitted by the soft palate and its pillars on the lateral pharyngeal walls and the base of the tongue, these vibrations give rise to traumatic lesions on these soft tissues as has been shown in numerous pathologico-anatomic studies; the characteristics of profound snorers do not differ qualitatively from those of apnea patients ${ }^{3,17}$.

Recent studies confirm that destabilization of the pharynx can be a result of deterioration of its mucosal ${ }^{11}$, muscular $^{14}$, and/or neural ${ }^{2,13,14}$ components of either or both sides, sensory or motor, of the mechanism that controls the geometric effects of the inspiratory gradient on the pharyngeal lumen. Accordingly, electromyography analyses show that chronic partial enervation of the palatopharyngeal muscle can occur during episodes of $\mathrm{OSA}^{5,14}$. In short, structural changes, side effects of the vibration trauma related to snoring, can affect every component of the pharyngeal complex, and contribute to collapse of this segment of the airways.

It is interesting to note that cerebral imagery of adults afflicted with $\mathrm{OSA}^{8,10}$ has pictured morphological changes of the grey matter affecting regions like the cerebellum that are vitally important in the initiation of inspiration ${ }^{8}$. Similarly, a recent study has shown metabolic alterations at the level of the hippocampus and the frontal cortex in children suffering from OSA in association with neuropsychic deficits that suggest a possible neural lesion related to OSA ${ }^{5}$.

However, it is important to note that the improvement in upper airway answer that children display after OSA treatment ${ }^{9}$ gives indirect support to the concept asserting that central nervous system alterations are a result of, not a cause of OSA. If this view is correct, early diagnosis and treatment of OSA in children should protect their neurological functioning. The conclusion to be drawn from all these studies is the importance of etiological treatment of the cause of snoring: the breathing disorders of nasal airways and their consequences on the inspiratory airflow through the pharynx. 


\section{3 - CONCLUSION}

Today we are justified in drawing the simple, clear, eloquent, even enthusiastic conclusion that orthodontic specialists occupy a privileged position for participating in the etiological treatment of OSA. By resorting to their unique technical capabilities of making shapes as functional as possible that would be definitively compromised if they did not take action. In others words, by reshaping facial structures that, untreated, would cause a profound deterioration of the quality of life of their patients and expose them to complications that could put at stake their prognosis for life. "Adaptive" palatal expansion, which is capable of freeing the pharynx from the suffocating constraints imposed by constricted nasal valves and nasal fossa, is truly an intervention that can deliver etiological correction. When this procedure is accomplished at a sufficiently early stage in patients' growth and development, OSA will prove to have been only a minor, if unpleasant, episode in the agitated functioning of a pharynx that may be able not to bear the permanent scare.

Assuredly, by gaining an understanding of OSA we are introduced to the full expanse of the functional complex with which the face is associated. "Smileology" has become a neologism thoroughly out of date. The vision of orthodontics stipulating that the treatment possibilities orthodontics can deliver would be limited to placing the teeth and their supporting dental arches in the best possible position has passed into oblivion. The same is true of the concept that surgical correction of cleft lips and palates has a simple objective of achieving a superficial improvement of the appearance of the nostrils and the uvula at the end of the first intervention. In all of these contexts, experience has shown that, over and above all restrictions, by enlarging the initial objective to optimizing the respiratory function of the face, the orthopedic as well as the surgical results achieve an indisputable gain in quality. We know that from now on the common therapeutic objective of the inter-disciplinary team of practitioners has been formally identified: it is to optimize the development of the faces of their patients with contributions that both dentofacial orthopedics and surgical correction of clefts can make. It is by acting directly to improve the breathing capacities of their patients that practitioners can contribute indirectly to ameliorating their cardiovascular and neuropsychological functioning. This objective fully justifies practitioners' personal investment in long and demanding educational programs. By reaching a new, more inclusive educational goal, they will derive the shared satisfaction of fulfilling their public health mission; and as doctors of dentofacial orthopedics they will thereby arrive at maturity after having for so many years hesitated to recognize the veritable dimensions of their calling.

René Dubos's advice "Think globally, act locally" certainly reflects the wisdom of a sage. 


\section{REFERENCES}

1. Bao G, Guilleminault C. Upper airway resistance syndrome: one decade later. Curr Opin Pulm Med 2004;10:461-7.

2. Boyd JH, Petrof BJ, Hamid Q, Fraser R \& Kimoff RJ. Upper airway muscle inflammation and denervation changes in obstructive sleep apnea. Am J Respir Crit Care Med 2004;170:541-6.

3. Edstrom L, Larsson $H$, Larsson L. Neurogenic effects on the palatopharyngeal muscle in patients with obstructive sleep apnoea: a muscle biopsy study. J Neurol Neurosurg Psychiatry 1992;55:916-20.

4. Guilleminault $C$, Huang YS, Kirisoglu C, et al. Is obstructive sleep apnea syndrome a neurological disorder? A continuous positive airway pressure follow-up study. Ann Neurol 2005;58:880-7.

5. Halbower AC, Degaonkar M, Barker PB, et al. Childhood obstructive sleep apnea associates with neuropsychological deficits and neuronal brain injury. PLoS Med 2006;3:e301.

6. Halbower AC, Ishman SL, McGinley BM. Breathing Childhood Obstructive SleepDisordered. Chest 2007;132:2030-41.

7. Macey PM, Henderson LA, Macey KE, et al. Brain morphology associated with obstructive sleep apnea. Am J Respir Crit Care Med 2002;166:1382-7.

8. Macey PM, Woo MA, Macey KE, et al. Hypoxia reveals posterior thalamic, cerebellar, midbrain and limbic deficits in congenital central hypoventilation syndrome. J Appl Physiol 2004;98:958-69.

9. Marcus CL, Katz ES, Lutz J, et al. Upper airway dynamic responses in children with the obstructive sleep apnea syndrome. Pediatr Res 2005;57:99-107.

10. Morrell MJ, McRobbie DW, Quest RA, et al. Changes in brain morphology associated with obstructive sleep apnea. Sleep Med 2003;4:451-4.

11. Paulsen FP, Steven P, Tsokos M, Jungmann K, Müller A, Verse T, Pirsig W. Upper airway epithelial structural changes in obstructive sleep-disordered breathing. Am J Respir Crit Care Med 2002;166:501-9.

12. Praud JP, Dorion D. Obstructive sleep disordered breathing in children: beyond adenotonsillectomy. Pediatr Pulmonol 2008;43:837-43.

13. Saboisky JP, Butler JE, McKenzie DK, Gorman RB, Trinder JA, White DP, Gandevia SC. Neural drive to human genioglossus in obstructive sleep apnoea. J Physiol 2007; 585.1:135-46.

14. Svanborg E. Impact of obstructive apnea syndrome on upper airway respiratory muscles. Respir Physiol Neurobiol 2005;147:263-72.

15. Talmant J, Rouvre M, Thibult JL et al. Contribution à l'étude des rapports de la ventilation avec la morphogenèse craniofaciale. Déductions thérapeutiques concernant I'ODF. Orthod Fr 1982;53:7-181.

16. Talmant J, Renaudin S, Renaud P. Ventilation et mécanique de I'oro-pharynx. Rev Orthop Dento-faciale 1998;32:105-66.

17. Woodson BT, Garancis JC, Toohill RJ. Histopathologic changes in snoring and obstructive sleep apnea syndrome. Laryngoscope 1991;101:1318-22. 